\title{
Recent advances in exacerbations of asthma
}

\section{Annemarie Sykes, ${ }^{1}$ Terence Seemungal, ${ }^{2}$ ICEAD contributors}

The major morbidity, mortality and health care costs associated with asthma are related to exacerbations. In order to reduce this massive healthcare burden, the prevention and treatment of exacerbations needs to be a greater focus for research. The following review is based on the presentations made at the first International Congress on Exacerbations of Airway Disease and summarises the current state of research on acute exacerbations of asthma.

The lack of a universally accepted definition for an exacerbation of asthma creates difficulties for interpretation of clinical research and trial results. Recently, an ERS/ATS task force addressed this, and to encourage uniformity defined moderate and severe exacerbations based on the actions of the patient and physician rather than symptoms (unpublished information). These definitions are a step forward for trial design and interpretation but it is acknowledged that no single definition will fit all clinical research needs.

\section{EXACERBATION AETIOLOGY}

Approximately $80 \%$ of asthma exacerbations are associated with respiratory viral infections, and rhinoviruses (RV) are responsible for about two-thirds of these. ${ }^{1}$ Despite the importance of viral infections, a combination of interacting factors is likely to be involved. A synergistic interaction between allergen sensitisation, allergen exposure and virus infection has been detected in adult patients with asthma during acute exacerbations. Sensitised, exposed and infected patients had a significantly increased risk of

\footnotetext{
${ }^{1}$ Department of Respiratory Medicine, National Heart and Lung Institute, MRC and Asthma UK Centre in Allergic Mechanisms of Asthma, Imperial College London, London, UK; ${ }^{2}$ Department of Clinical Medical Sciences, University of West Indies, Mount Hope, Trinidad and Tobago
}

Correspondence to: Dr Annemarie Sykes, Department of Respiratory Medicine, National Heart and Lung Institute, MRC and Asthma UK Centre in Allergic Mechanisms of Asthma, Imperial College London, Norfolk Place, London W21PG, UK; Annemarie.sykes@ imperial.ac.uk admission for exacerbations. ${ }^{2}$ An even greater interaction exists in children. ${ }^{3}$

The role of RV in exacerbations has been investigated in a human experimental model in patients with mild asthma. During acute infection, patients with asthma had increased lower respiratory tract symptoms, falls in lung function and increases in bronchial hyperresponsiveness compared with those without asthma. Virus load correlated strongly with asthma symptoms and hyperresponsiveness, implicating severity of infection as the main determinant of exacerbation severity (data submitted for publication). Interferon $\beta$ production in response to RV is known to be reduced in patients with asthma. ${ }^{4}$ In this model, deficiencies of a new class of interferons, type III/ $\lambda$ interferons, was reported and this was related to clinical outcome. ${ }^{5}$

Investigation into mechanisms of exacerbations has been boosted by the recent development of a small animal model of rhinovirus infection. ${ }^{6}$ This provides an important tool to investigate mechanisms of exacerbations and for testing new treatments. Another alternative for studying exacerbations is the human allergen inhalation challenge model. This induces an allergic asthmatic exacerbation by inhalation of allergens directly into the airway, used to date in over 450 individuals with mild allergic asthma.'

Although viral infections are associated with the great majority of exacerbations, there is increasing evidence that atypical bacterial infections, such as Chlamydophila pneumoniae, may also be important. There is a relationship between $C$ pneumoniae specific IgA and exacerbation frequency, ${ }^{8}$ and in one study $38 \%$ of adults attending the emergency room with an asthma exacerbation had an increase in $C$ pneumoniae antibody levels. ${ }^{9}$

The TELICAST trial was a double blind, placebo controlled study which randomised adults with asthma exacerbations to the ketolide antibiotic telithromycin or placebo. ${ }^{10}$ A significantly greater improvement in asthma symptoms and lung function from exacerbation to end of treatment was seen in the telithromycin group. This treatment effect may be the result of treating atypical infection and/or anti-inflammatory properties of telithromycin. Further studies are required to see whether there are similar benefits with related macrolide antibiotics.

\section{PREDICTING AND PREVENTING EXACERBATIONS}

The ability to predict an exacerbation or identify patients with asthma at risk would be clinically useful.

Exacerbations in children and young adults peak in September; this is reproduced in multiple northern hemisphere countries, is earliest in 6 year old and is related to school return. In children investigated during the peak, $62 \%$ of cases and $41 \%$ of controls had evidence of viral infection, predominantly RV (84\%), highlighting viral aetiology as important. ${ }^{11}$

In a US study, the risk of hospitalisation was increased if a patient had been hospitalised previously, was African American or Hispanic, had a low household income or was exposed to tobacco smoke. African American and Hispanic patients with asthma were also at risk for increased mortality as were older patients, those with worse lung function and those exposed to tobacco smoke. ${ }^{12}$

Detecting signs of an exacerbation early would enable administration of treatment and ideally prevent it from occurring. Monitoring sputum eosinophils is a theoretical possibility for early detection; when used to guide anti-inflammatory treatment, this strategy increased time to exacerbation and decreased total exacerbation number. Exhaled nitric oxide (eNO), a surrogate marker for eosinophillic inflammation, has also been investigated in the context of predicting exacerbations. Patients with asthma that had inhaled corticosteroid (ICS) treatment withdrawn were followed-up until an exacerbation occurred. eNO measurements were made regularly until this point and were raised both at exacerbation and 1 week previously. ${ }^{13}$

Peak flow (PEF) measurements have traditionally been used to predict an exacerbation. However, PEF drops occur almost simultaneously with exacerbation onset. The utility of PEF for predicting poor asthma control has been advanced by subjecting multiple PEF measurements to fluctuation analysis. Using this it is possible to predict the risk to the individual of exacerbation in the subsequent month. ${ }^{14} 15$ However, despite using the term exacerbation, in reality this method 
predicts "loss of control" or "PEF instability" rather than an acute exacerbation.

\section{PATHOGENESIS}

Expiratory flow limitation during an exacerbation is secondary to a combination of bronchospasm, airway inflammation and increased mucus inspissation. Acute dynamic pulmonary hyperinflation occurs because of air trapping which, if sustained, weakens and overloads the inspiratory muscles and compromises gas exchange.

Significant pulmonary hyperinflation is present even with relatively mild bronchoconstriction during methacholine challenge in patients with asthma and contributes to perceived respiratory difficulty. ${ }^{16}$

The cellular mechanisms of exacerbations have been investigated mainly by sputum sample analysis. Quantitative sputum cell counts provide the most comprehensive, specific and discriminative information. In stable asthma the majority have normal sputum cell differentials, with only $10 \%$ having predominantly eosinophilic or neutrophilic sputum. During an exacerbation, only $15 \%$ are normal with $35 \%$ eosinophilic, $25 \%$ neutrophilic and 10\% having a mixed eosinophilic and neutrophilic picture. The rest were unclassifiable or unsuitable for analysis. Interestingly, in $50 \%$ of individuals, successive exacerbations had different types of inflammation. ${ }^{17}$

Sputum cell counts were used in a controlled trial of patients with asthma randomised to therapy adjusted according to guidelines or to sputum results. Patients with sputum based treatment had significantly fewer exacerbations, the largest reduction being in eosinophilic exacerbations. Time to first exacerbation was prolonged, lower total amounts of ICS and long acting beta agonists were needed and treatment costs were lower. ${ }^{17}$

The main cellular inflammatory response to virus induced exacerbations is neutrophilic. In naturally occurring colds, patients with asthma have an increased proportion and absolute number of neutrophils, associated with high levels of the neutrophil chemoattractant interleukin 8, and levels strongly correlate with sputum neutrophils. ${ }^{18}$ Eosinophils are also thought to be involved in virus induced asthma as eosinophil cationic protein levels are also raised.

There is currently great interest in using biomarkers to monitor asthma control with the aim of reducing exacerbation frequency. eNO is quick and easy to perform compared with monitoring airway hyperresponsiveness or sputum eosinophils. Previous studies using eNO to adjust asthma therapy have reported a marginal decrease in exacerbations, and a recent study reported that eNO monitoring allowed a substantial decrease in inhaled corticosteroids with no increase, and possibly a decrease, in exacerbation frequency. ${ }^{19}$

\section{CURRENT TREATMENTS}

How good are current treatments in preventing exacerbations? The FACET study demonstrated that the combination of long acting beta agonists and ICS reduced exacerbations, especially severe exacerbations, in comparison with ICS alone. $^{20}$ In the START study, ICS decreased exacerbations by $25 \%$ in newly diagnosed patients with mild asthma, and patients had fewer courses of systemic corticosteroids and more symptom free days than with placebo. ${ }^{21}$

Treating exacerbations was also the aim of the Paediatric Asthma Controller Trial (PACT). Three types of controller medication were investigated: low dose ICS, fluticasone with salmeterol (PACT combination) or oral montelukast, with more exacerbations in those on the oral leukotriene antagonist and less on ICS. ${ }^{22}$

Leukotriene receptor antagonists also prevent exacerbations; zafirlukast reduced exacerbation frequency in adults with mild asthma ${ }^{23}$ and montelukast added to usual therapy in children during the September peak. ${ }^{24}$

Anti-IgE treatment decreased exacerbation frequency, impacting both the number of subjects who developed exacerbations and duration of exacerbation. $^{25}$

The strategy of using formoterol/budesonide as both preventer and reliever has also been shown to reduce exacerbations, resulting in prolonged time to first, second and third exacerbation, improved symptoms and a reduced severe exacerbation rate. ${ }^{2627}$ An as required combination of beclomethasone/albuterol also reduced total steroid use and number of exacerbations. $^{28}$

\section{FUTURE TREATMENTS}

More therapeutic options are needed as these clinical trials show that current treatments can prevent, at best, $50 \%$ of exacerbations and this figure may be lower in patients not enrolled in clinical trials. ${ }^{29}$ Better therapies are particularly required for exacerbations that are predominantly neutrophilic or associated with viruses.
Vaccination for RV is not feasible because of the large number of serotypes and no licensed new antiviral therapy is available.

Modulation of the host response to virus infection is a potential approach to therapy that would have the advantage of modifying all virus associated exacerbations. The interferon deficiencies observed in asthma ${ }^{45}$ indicate interferons could be developed as a therapy.

Novel anti-inflammatory treatments are another area of interest. Macrolides have anti-inflammatory effects; in vitro erythromycin reduces interleukin 8, tumour necrosis factor $\alpha$, interleukin 6 and interleukin $1 \beta$, and the related ketolide antibiotic has clinical efficacy in the treatment of exacerbations in adults. ${ }^{10}$ Similar trials with macrolides are now needed.

\section{FUTURE DIRECTIONS}

Virus infections are critically important in exacerbations of asthma, and interactions between viruses, other pathogens and allergen exposure need further study. Research should also address the role of susceptibility factors such as deficient interferon responses, the roles of oxidative stress, transcription factors and signalling pathways, and the importance of different genetic backgrounds.

There is potential in the future to define different exacerbation phenotypes based on pathological findings which will influence the design of future clinical trials; however, this is complicated because the inflammatory profile frequently changes in individual patients during subsequent exacerbations.

Future research needs to have a greater focus on the prevention and treatment of exacerbations before the morbidity, mortality and healthcare costs can be reduced.

Funding: The organisers would like to acknowledge the Macrae group for their support of and their organisation of this meeting.

\section{Competing interests: None.}

- A list of the International Congress on Exacerbations of Airway Disease (ICEAD) contributors is published online only at http://thorax.bmj.com/content/vol63/issue9

Thorax 2008;63:758-760. doi:10.1136/thx.2008.099036

\section{REFERENCES}

1. Corne JM, Marshall C, Smith S, et al. Frequency, severity, and duration of rhinovirus infections in asthmatic and non-asthmatic individuals: a longitudinal cohort study. Lancet 2002;359:831-4.

2. Green RM, Custovic A, Sanderson G, et al. Synergism between allergens and viruses and risk of hospital admission with asthma: case-control study. BMJ 2002;324:763

3. Murray CS, Poletti G, Kebadze T, et al. Study of modifiable risk factors for asthma exacerbations: virus 
infection and allergen exposure increase the risk of asthma hospital admissions in children. Thorax 2006;61:376-82.

4. Wark PAB, Johnston SL, Bucchieri F, et al. Asthmatic bronchial epithelial cells have a deficient innate immune response to infection with rhinovirus. J Exp Med 2005;201:937-47.

5. Contoli M, Message SD, Laza-Stanca V, et al. Role of deficient type III interferon-[lambda] production in asthma exacerbations. Nat Med 2006;12:1023-6.

6. Bartlett NW, Walton RP, Edwards MR, et al. Mouse models of rhinovirus-induced disease and exacerbation of allergic airway inflammation. Nat Med 2008;14:199-204.

7. BouletL-P, Gauvreau G, Boulay M-E, et al. The allergen bronchoprovocation model: an important tool for the investigation of new asthma anti-inflammatory therapies. Allergy 2007;62:1101-10.

8. Cunningham AF, Johnston SL, Julious SA, et al. Chronic Chlamydia pneumoniae infection and asthma exacerbations in children. Eur Respir J 1998;11:345-9.

9. Wark PAB, Johnston SL, Simpson JL, et al. Chlamydia pneumoniae immunoglobulin A reactivation and airway inflammation in acute asthma. Eur Respir $J$ 2002;20:834-40.

10. Johnston SL, Blasi F, Black PN, et al. The effect of telithromycin in acute exacerbations of asthma. N Engl J Med 2006;354:1589-600.

11. Johnston NW, Johnston SL, Duncan JM, et al. The September epidemic of asthma exacerbations in children: A search for etiology. J Allergy Clin Immunol 2005;115:132-8.

12. Boudreaux ED, Emond SD, Clark S, et al. Acute asthma among adults presenting to the emergency department: the role of race/ethnicity and socioeconomic status. Chest 2003;124:803-12

13. Jones SL, Kittelson J, Cowan JO, et al. The predictive value of exhaled nitric oxide measurements in assessing changes in asthma control. Am J Respir Crit Care Med 2001;164:738-43.

14. Frey U, Brodbeck T, Majumdar A, et al. Risk of severe asthma episodes predicted from fluctuation analysis of airway function. Nature 2005;438:667-70.

15. Hacking D, Knight JC, Rockett K, et al. Increased in vivo transcription of an IL-8 haplotype associated with respiratory syncytial virus disease-susceptibility. Genes Immun 2004:5:274-82.

16. Lougheed MD, Fisher T, O'Donnell DE. Dynamic hyperinflation during bronchoconstriction in asthma: implications for symptom perception. Chest 2006;130:1072-81.

17. Jayaram L, Pizzichini MM, Cook RJ, et al. Determining asthma treatment by monitoring sputum cell counts: effect on exacerbations. Eur Respir J 2006;27:483-94.

18. Pizzichini M, Pizzichini E, Eftthimiadis A, et al. Asthma and natural colds. Inflammatory indices in induced sputum: a feasibility study. Am J Respir Crit Care Med 1998;158:1178-84.

19. Smith AD, Cowan JO, Brassett KP, et al. Use of exhaled nitric oxide measurements to guide treatment in chronic asthma. N Engl J Med 2005;352:2163-73.

20. Pauwels RA, Lofdahl CG, Postma D, et al. Effect of inhaled formoterol and budesonide on exacerbations of asthma. N Engl J Med 1997;337:1405-11.

21. Pauwels RA, Pedersen S, Busse WW, START Investigators Group. Early intervention with budesonide in mild persistent asthma: a randomised, double-blind trial. Lancet 2003;361:1071-6.
22. Sorkness CA, Lemanske RF, Mauger D, et al. Long term comparison of 3 controller regimes for mildmoderate persistent childhood asthma: The paediatric asthma controller trial. J Allergy Clin Immunol 2007;119:64-72.

23. Barnes NC, Miller CJ. Effect of leukotriene receptor antagonist therapy on the risk of asthma exacerbations in patients with mild to moderate asthma: an integrated analysis of zafirlukast trials. Thorax 2000;55:478-83.

24. Johnston NW, Mandhane PJ, Dai J, et al. Attenuation of the September epidemic of asthma exacerbations in children: a randomized, controlled trial of montelukast added to usual therapy. Pediatrics 2007; 120:e702-12.

25. Rees PJ. Omalizumab reduces exacerbation and steroid use in chronic asthma. Arch Dis Child Ed Pract 2007:92:ep127.

26. O'Byrne PM, Bisgaard H, Godard PP, et al. Budesonide/formoterol combination therapy as both maintenance and reliever medication in asthma. $A m \mathrm{~J}$ Respir Crit Care Med 2005;171:129-36.

27. Rabe KF, Atienza T, Magyar P, et al. Effect of budesonide in combination with formoterol for reliever therapy in asthma exacerbations: a randomised controlled, double-blind study. Lancet 368:744-53.

28. Papi A, Canonica GW, Maestrelli $P$, et al. Rescue use of beclomethasone and albuterol in a single inhaler for mild asthma. $N$ Engl J Med 2007;356:2040-52.

29. Rabe KF, Vermeire PA, Soriano JB, et al. Clinical management of asthma in 1999: the Asthma Insights and Reality in Europe (AIRE) study. Eur Respir $J$ 2000;16:802-7. 